\title{
Desvenlafaxine for the Acute Treatment of Depression: A Systematic Review and Meta-analysis
}

\author{
Authors \\ Affiliations \\ Key words \\ - depression \\ - desvenlafaxine \\ - SNRI \\ - review \\ meta-analysis
}

\section{Z. G. Laoutidis ${ }^{1}$, K. T. Kioulos ${ }^{2}$}

Department of Psychiatry and Psychotherapy, Medical Faculty of the Heinrich Heine University, Düsseldorf, Germany Eginition Hospital, ${ }^{\text {st }}$ Department of Psychiatry, Medical Faculty, University of Athens, Vasilissis Sofias Avenue, Athens, Greece

$\begin{array}{lr}\text { received } & 13.03 .2015 \\ \text { revised } & 10.05 .2015 \\ \text { accepted } & 10.06 .2015\end{array}$

Bibliography

DOI http://dx.doi.org/

$10.1055 / \mathrm{s}-0035-1555879$

Published online:

July 23, 2015

Pharmacopsychiatry 2015;

48: 187-199

(c) Georg Thieme Verlag KG

Stuttgart · New York

ISSN 0176-3679

\section{Correspondence}

Zacharias G. Laoutidis

Department of Psychiatry and Psychotherapy

Medical Faculty of the Heinrich

Heine University

Bergische Landstrasse 2

40629 Düsseldorf

Germany

laoutidis@hotmail.com

\section{Abstract}

$\nabla$

Introduction: Desvenlafaxine, the active metabolite of venlafaxine, was approved in 2008 by the FDA for the treatment of depression. The aim of the present review is to provide an overview of the existing trials with desvenlafaxine and assess its overall efficacy and tolerability.

Methods: We searched in PubMed, EMBASE and the Cochrane Library for eligible studies (double-blind randomized control trials). A random effects model was used for the estimation of effect sizes.

Results: 17 trials were found in total. In the placebo-controlled trials the overall risk ratio

\section{Introduction}

\section{$\nabla$}

Desvenlafaxine is a relatively novel agent that was approved in 2008 in the USA for the treatment of major depressive disorder. It is the main metabolite of venlafaxine, a selective serotonin and norepinephrine reuptake inhibitor, which is considered to be one of the most effective antidepressants today [1]. Desvenlafaxine appears to share the same pharmacodynamic properties as the parent substance [2]. The efficacy of active metabolites is not self-evident and should not be taken for granted; for example, the active metabolite of clozapine - the most effective antipsychotic drug [3] - was not found to be effective in the treatment of schizophrenia. Several trials have been conducted so far on the efficacy of desvenlafaxine in the treatment of major depressive disorder and an early meta-analysis showed significant results in both primary (HAM- $\mathrm{D}_{17}$ scores) and secondary (response and remission rates) outcomes [4]. The efficacy of desvenlafaxine has been tested further in more recent studies, thus making it imperative to update the first review. The objective of our review is to give an overview of the existing literature; we focus solely on clini- for response was $1.24(1.16-1.32, \mathrm{p}<0.001)$, for remission $1.29(1.16-1.43, \mathrm{p}<0.001)$, for dropouts $1.16(0.99-1.35, \mathrm{p}=0.066)$ and for dropouts due to adverse events 1.98 (1.45-2.69, $\mathrm{p}<0.001)$. There were no differences between the various doses that were used (i.e., $50 \mathrm{mg}$, $100 \mathrm{mg}, 200 \mathrm{mg}, 400 \mathrm{mg}$ ). The mean risk ratio for response in the head-to-head trials was 0.90 (0.82-0.98, $\mathrm{p}=0.014)$ and for remission 0.82 (0.71-0.95, $\mathrm{p}=0.009)$.

Discussion: The risk ratios for response and remission were moderate. We further provide some evidence that desvenlafaxine might not be as efficacious as other antidepressants.

cally relevant parameters for efficacy (response and remission rates) and tolerability (discontinuation rates and discontinuation due to adverse effects), and we will estimate effect sizes for various doses with the aim of detecting a possible dose-dependent effect. Further, we compare desvenlafaxine with other antidepressant agents, if any head-to-head trials are available.

\section{Methods \\ $\nabla$}

\section{Search strategy}

The inclusion criteria for the studies were the following: Double-blind, randomized controlled trials (RCTs), either placebo-controlled or headto-head trials. We searched for studies in the electronic databases PubMed, EMBASE and the Central Register of Controlled Trials of the Cochrane Library. The only search term was "desvenlafaxine". The applied limits of the search were that the articles should have been published by December 31, 2014. We further searched through the reference lists of reviews and related articles to identify any additional studies. 


\section{Article selection and review strategy}

The selection of studies involved an initial screening of title and abstract in order to find studies fulfilling the above inclusion criteria. If it was not clear from the title or abstract that a study should be rejected, the full text was obtained. This process was conducted independently by both authors in order to reduce the possibility of rejecting relevant articles.

The data were extracted independently by both authors. In case of disagreement, a clinician experienced in psychopharmacology could be consulted to mediate consensual decisions. Dichotomous data (rates for response and remission) were collected for the primary outcomes of this review. Secondary outcomes were the risk of dropouts due to any reason and the risk of dropouts due to adverse effects.

\section{Statistical methods (meta-analysis)}

Meta-analysis was performed when more than one trial was available in either group of studies (placebo-controlled and head-to-head trials). A random-effects model was applied because of the assumption that the true effect size was not the same in all studies. Relative risk ratios (RR) were computed for dichotomous data, because they have the advantage of being more intuitive than odds ratios (OR). A significant proportion of meta-analyses use the odds ratio as the main effect size; in order to make our results comparable with the results from other studies we also estimated the OR for response, remission and discontinuation. Values for RR and OR greater than 1 mean that desvenlafaxine is superior over placebo or the compared antidepressant (and vice versa for values under 1). In estimating risk ratios for response and remission, we accepted the recommendation of the Cochrane Handbook for systematic reviews, that if data from the intention-to-treat population are not reported, an available case analysis is the best alternative [5]. In the case of unusable data (e.g., analysis per protocol) the study was excluded at first from our main analysis and sensitivity analysis was performed afterwards in order to evaluate the impact of the trial on the overall effect size.

In the case of zero events trials (in one or in both arms), the standard continuity correction of 0.5 was applied [6]. If data were not provided in the article or were reported in a non-useful way, the corresponding authors were contacted. When this approach was unfruitful, we proceeded as follows: a) we searched in previous reviews and reports for suitable data, b) when data were reported as proportions, we converted them back to natural numbers. If the result was unclear, the mean of the possible values was used in the main analysis (for example, if a group of 150 patients is reported to have $65 \%$ responders, the possible number of responders is 97 or 98 , in which case 97.5 was used in the main analysis). In order to ensure that this method did not have a significant impact on the results, we performed sensitivity analysis (first sensitivity analysis or SA-1) for the best case (highest number of the verum group and lowest number of the placebo group) and the worst case (exactly the opposite) scenario. c) We extracted data from graphs using the WebPlotDigitizer Version 3.3 [Ankit Rohatgi (2014), ZENODO, 10.5281 /zenodo.10532]. d) If graphs were not available, we converted continuous data to dichotomous by the method described by Furukawa et al. [7]. This method is applicable only for response rates, not for remission rates.

The calculations were performed using standard formulas in Microsoft Excel (Excel 2003 Edition, Microsoft, Redmond, WA) [8]. The forest plot was also created in Microsoft Excel according to a guide published by Neyeloff et al. [9]. Heterogeneity $\mathrm{I}^{2}$ was computed in order to assess the percentage of the overall variability attributed to between-study variability. The risk of bias in individual studies was evaluated using the Cochrane Collaboration's domain-based tool, which assesses allocation concealment, sequence generation, blinding, selective outcome reporting and other sources of bias. The risk of publication bias was assessed using a funnel plot and Egger's regression method [10].

\section{Results}

$\nabla$

\section{Search results}

The electronic searches provided 326 references from MEDLINE, 935 from EMBASE and 95 references (clinical trials) from the Cochrane Library. After the initial scanning of the abstracts a total of 20 reports remained. These reports were further screened and assessed for eligibility and 5 of them were rejected. The remaining 14 reports fulfilled the inclusion criteria for the review (see flow diagram in $\bullet$ Fig. 1). Details for each trial are presented in $\triangle$ Table 1 . The complete list of the assessed trials and the reasons for rejection appear in Appendix A.

11 reports with a total of 12 placebo-controlled trials qualified for our main analysis [11-21]. 2 of these were 3 -arm studies which included a group that received a dose of desvenlafaxine below $50 \mathrm{mg}$; these 2 groups were excluded from the main analysis and included in an additional sensitivity analysis (second sensitivity analysis or SA-2), since in daily practice desvenlafaxine is not used in a dose of $10 \mathrm{mg}$ or $25 \mathrm{mg}$. In addition, we found in 2 reviews an unpublished report with the code name Des 223, which was also included in the main analysis. 3 further placebocontrolled RCTs were identified [22-24]: 2 of them included only perimenopausal and postmenopausal women, while the third included only patients who were employed. These 3 last trials used a slightly different design: They recruited patients based on their MADRS score (a cutoff of 22 or 25), but estimated the response rates based on HAM-D scores in a subpopulation of the original sample, which had an initial HAM-D score above 18. Because of the different populations and study design, these 3 articles were used only in sensitivity analyses (third sensitivity analysis or SA-3).

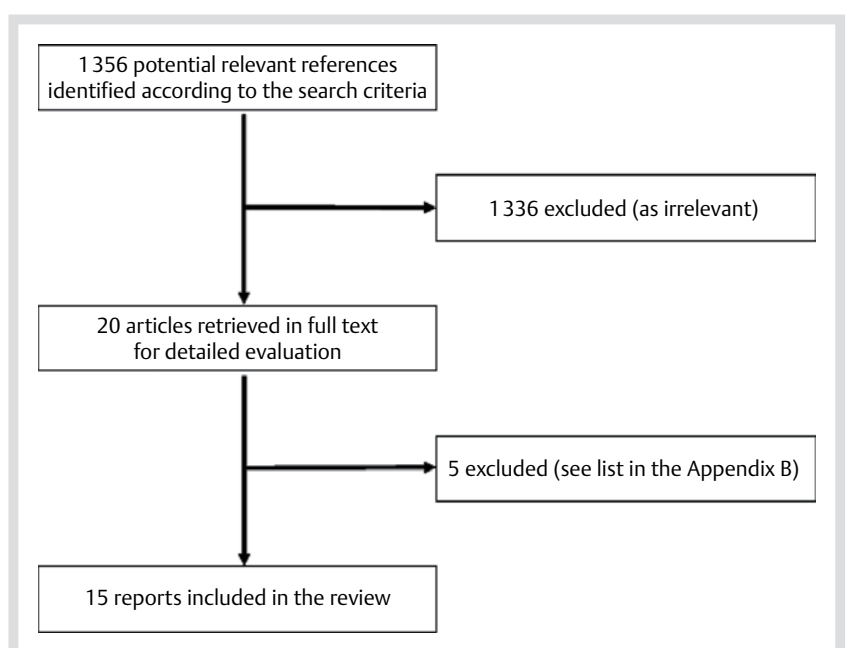

Fig. 1 Flow diagram of the study. 

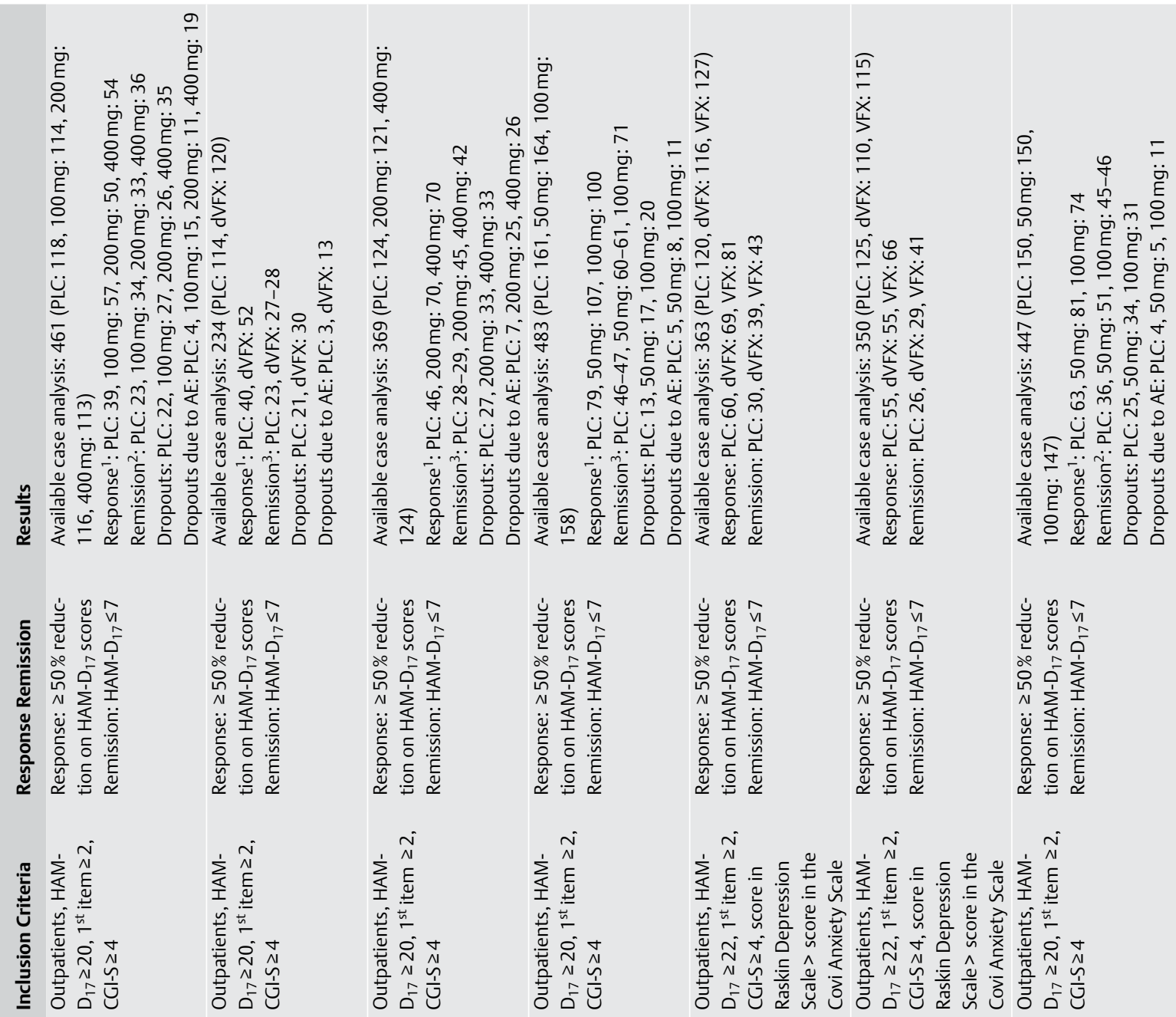

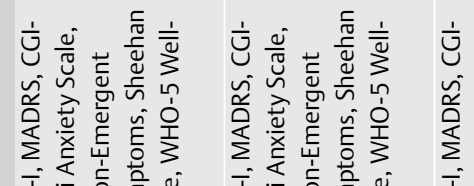

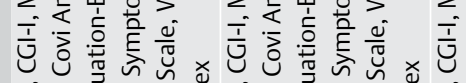

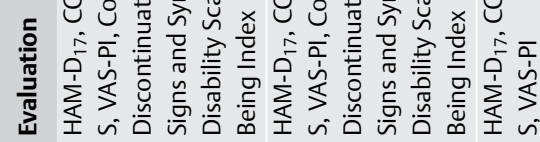

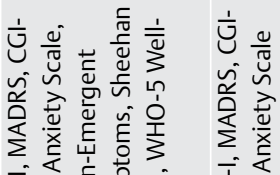

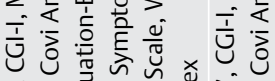

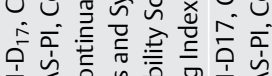

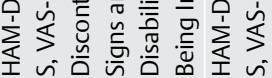

产宅 它

는 芩 윰

๖ั๊

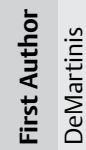

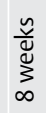

\&

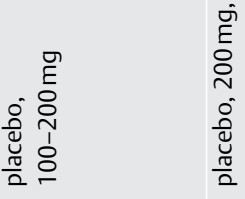

亏

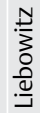

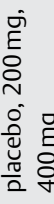

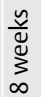

$\stackrel{n}{m}$

ڤे

产离 $\sum_{\infty}^{\frac{n}{d}}$

品

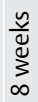

$\stackrel{ッ}{2}$

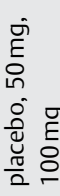

总

จั๊
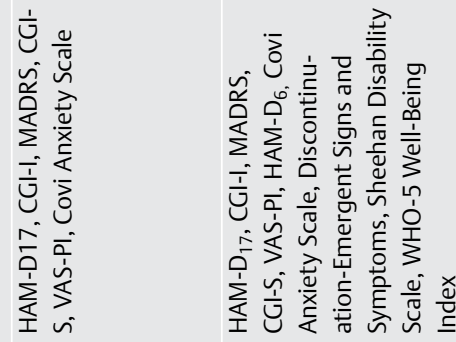

$\underset{\infty}{\tilde{\Phi}}$

$\frac{\infty}{2}$

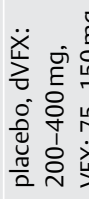

$\stackrel{\infty}{\circ} \mathrm{i}$

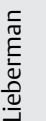




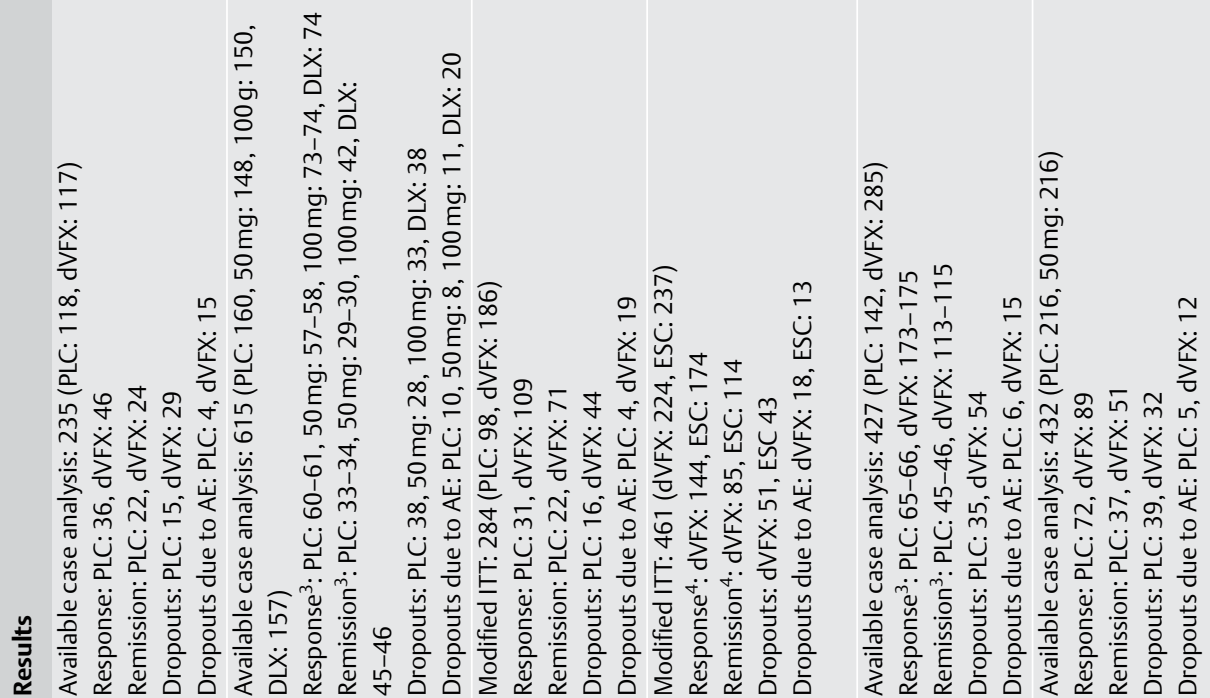

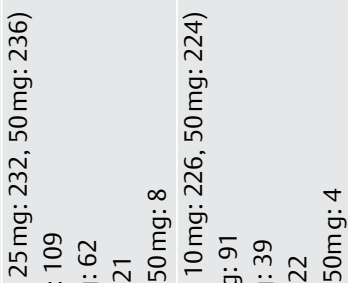

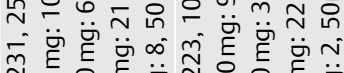

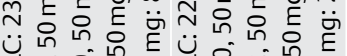

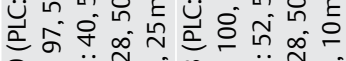

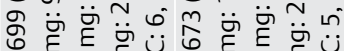

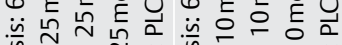

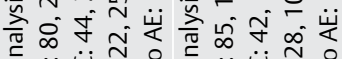
菂败

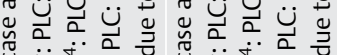

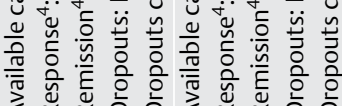

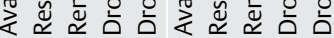

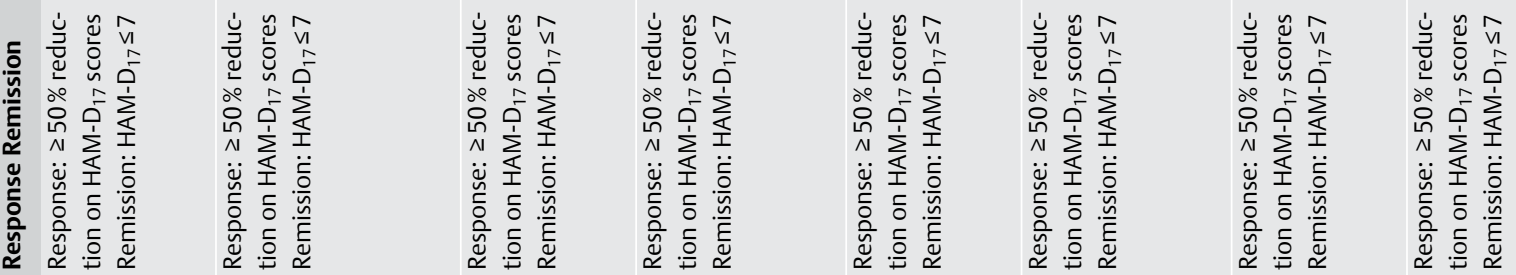
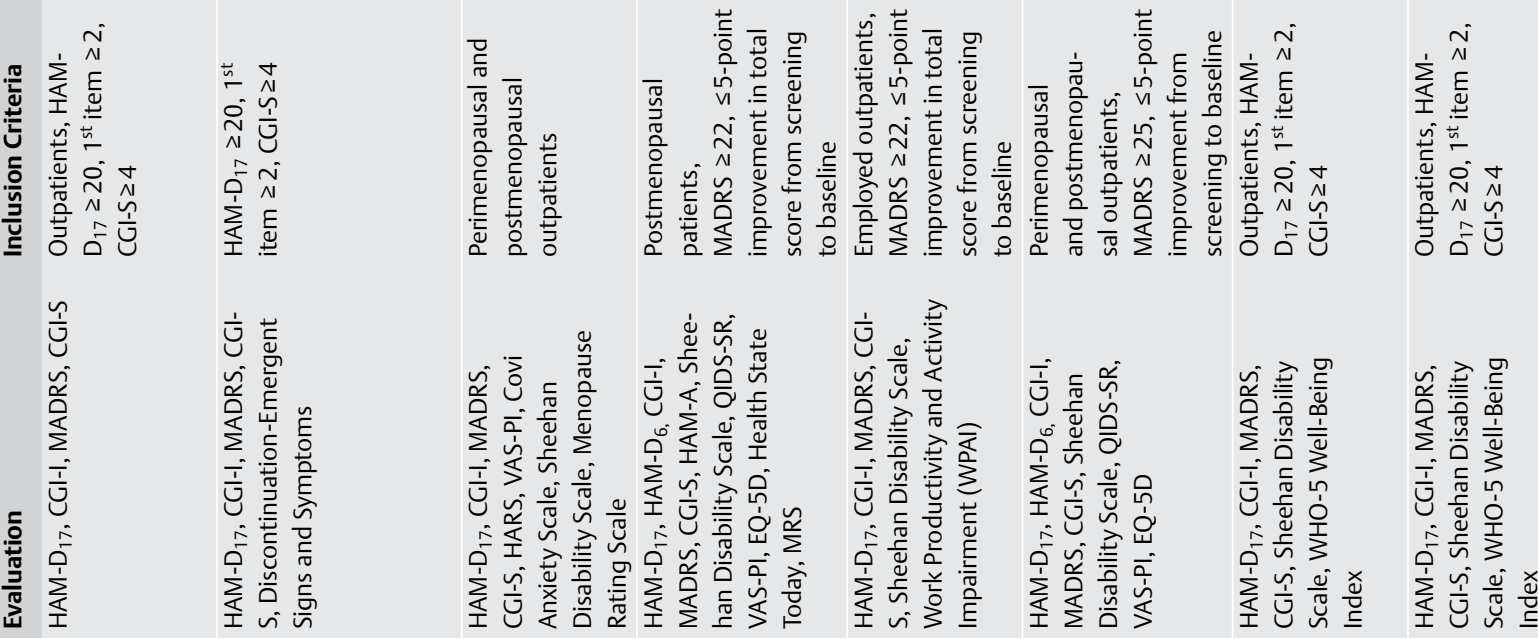

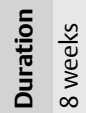

$\frac{n}{\frac{n}{\pi}}$

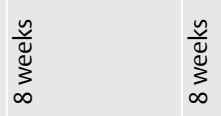

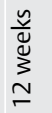

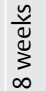

$\underset{\infty}{\check{\Psi}}$

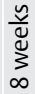

$2 \stackrel{\sim}{\sharp}$

$\infty$

$\stackrel{\infty}{m}$

ڤิ

$\hat{q}$

学

\&

$\approx$

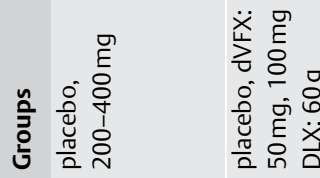

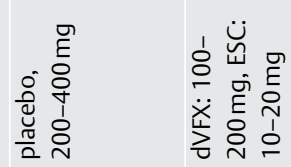

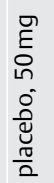

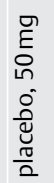

离 突

兽

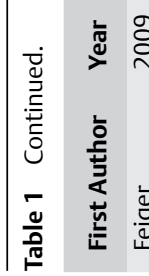

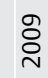

¿

$\stackrel{\circ}{\grave{2}}$

సे

$\stackrel{m}{n}$

ì

ڤั

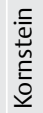

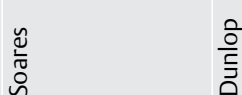

产

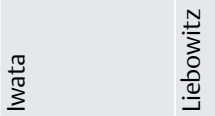




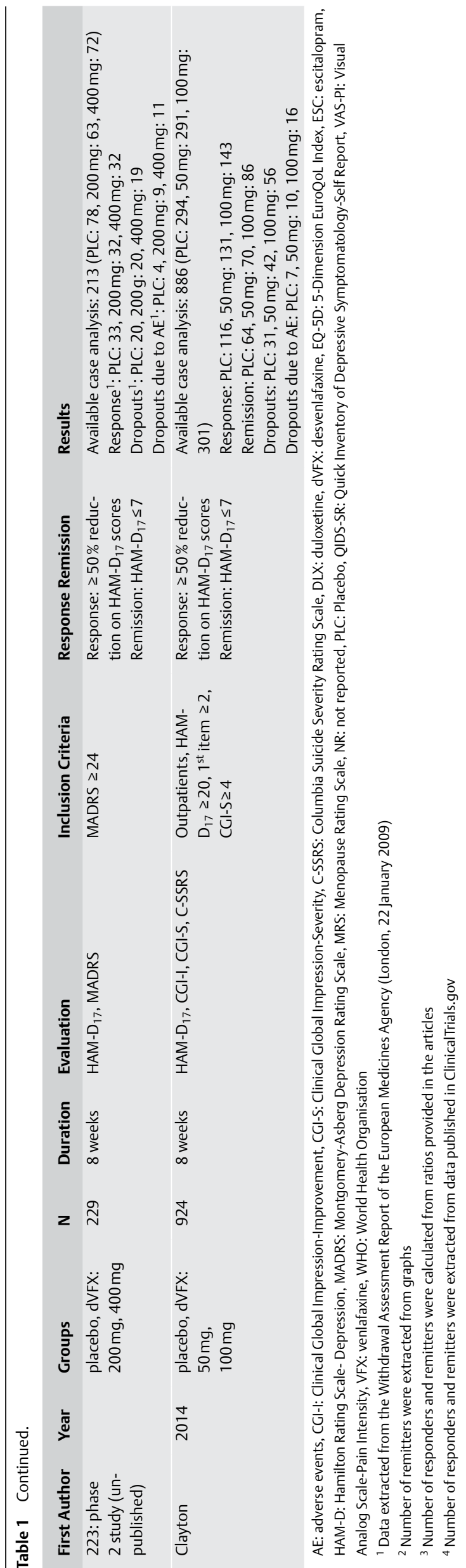

The exact numbers of responders in trials that were published before 2009 were provided in the official withdrawal assessment report of the European Medicine Agency. In the other cases, the reported proportions were used to estimate the number of responders as described in the methods section. In 2 cases the remission rates were not reported $[11,16]$; we extracted the data from the provided graphs using WebPlotDesigner.

A separate meta-analysis was performed with 4 head-to-head trials, which enabled a direct comparison of the efficacy of desvenlafaxine and other antidepressants. 2 of the above-mentioned reports (with a total of 3 trials) included an additional comparison group that received another antidepressant (venlafaxine in 2 cases and duloxetine in the third case) $[15,18]$. The third report included no placebo group and compared desvenlafaxine with escitalopram in peri- and postmenopausal women with depression [25].

\section{Meta-analysis \\ Effect size for efficacy}

In the main analysis the mean risk ratio for response was 1.24 (95\% CI: 1.16-1.32; $\mathrm{p}<0.001$ ) (० Fig. 2 ) and the mean risk ratio for remission was 1.29 (95\% CI: 1.16-1.43; $\mathrm{p}<0.001$ ). In our sensitivity analyses the relative risk ratios ranged between 1.23 and 1.26 for response and between 1.27 and 1.31 for remission. The results are presented in $\odot$ Table 2 .

\section{Efficacy of fixed doses and comparisons between them}

We estimated the risk ratios for response and remission for 4 separate doses ( 0 Table 3 ); all results were statistically significant. The risk ratio for remission in trials that used a flexible dose lacked statistical significance. The 4 separate doses were compared with each other; 2 direct comparisons and 4 indirect comparisons were performed, none of which were statistically significant ( $\odot$ Table 4,5).

\section{Tolerability}

12 trials were considered in the estimation of tolerability parameters. The overall risk ratio for discontinuation, based on the safety population of each study, was 1.16 (95\% CI: 0.99-1.35; $\mathrm{p}=0.066)$. The risk ratio for discontinuation due to adverse effects was 1.98 (95\% CI: $1.45-2.69 ; \mathrm{p}<0.001$ ). The estimated odds ratios were $1.20(95 \% \mathrm{Cl}: 0.99-1.44 ; \mathrm{p}=0.059)$ and 2.07 (95\% CI: 1.48-2.89; $\mathrm{p}<0.001$ ), respectively.

\section{Head-to-head trials}

3 comparisons in total were possible: desvenlafaxine against venlafaxine, against SSNRIs (i.e., venlafaxine and duloxetine), and against antidepressants in general (i.e., venlafaxine, duloxetine and escitalopram). The risk ratios for response and remission were statistically significant only in the third comparison in favor of the other antidepressants. All results are presented in $\odot$ Table 6.

\section{Heterogeneity}

The computed heterogeneity $\mathrm{I}^{2}$ was $0 \%$ in the main analysis for response (95\% CI: $0-34 \%$ ) and $0 \%$ in the main analysis for remission rates (95\% CI: $0-29 \%)$. In our sensitivity analyses the heterogeneity remained low. Here, the low heterogeneity can be attributed to the similar designs of the studies included in the analysis and the homogeneity of the studied population. 


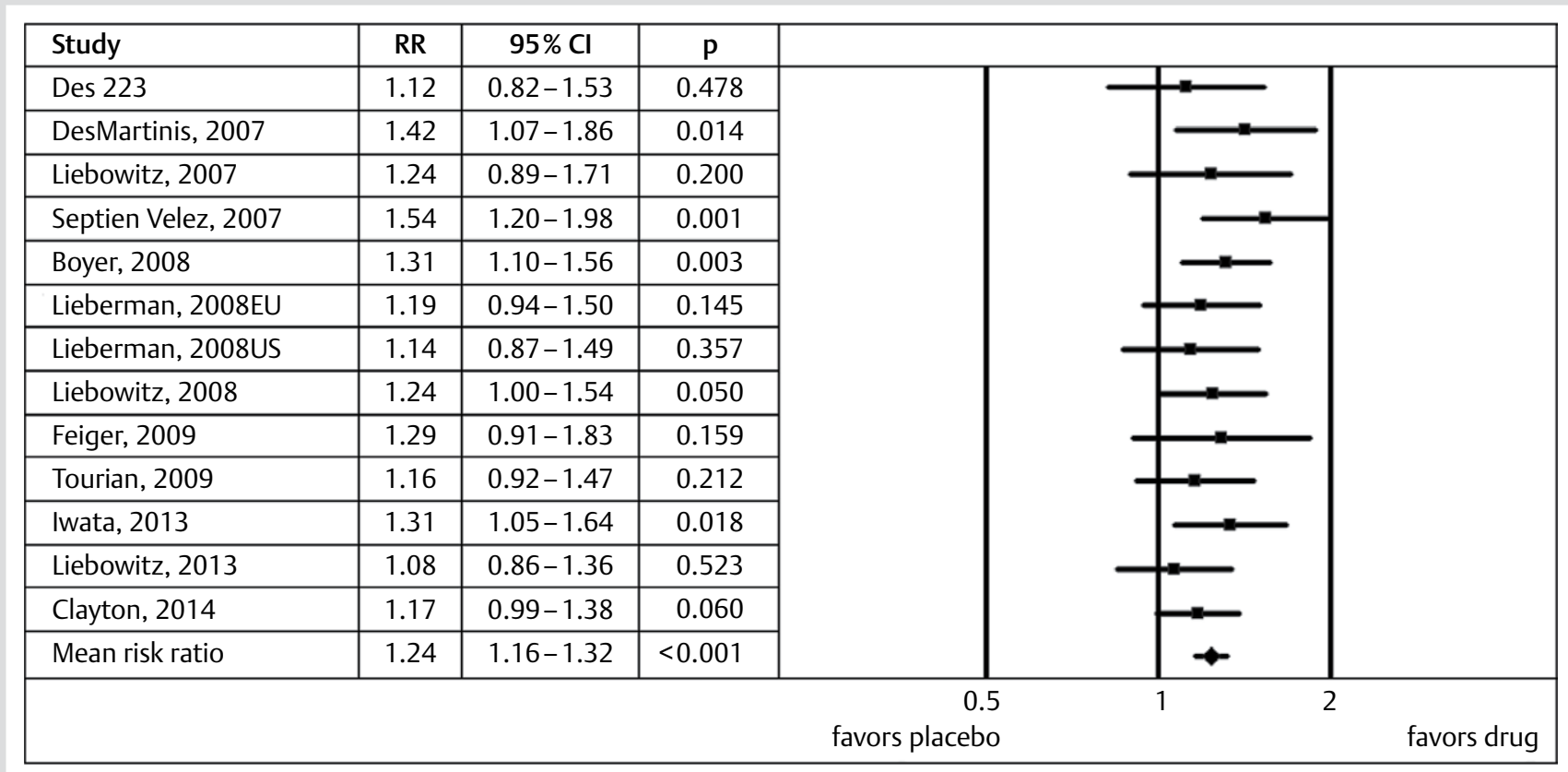

Fig. 2 Forest plot for risk ratios for response.

Table 2 Main analysis and sensitivity analyses for efficacy.

$\begin{array}{lllccccc}\text { Analysis } & \text { Dose range } & \mathbf{N} & \text { Effect size for response } & \mathbf{P} & \mathbf{N} & \text { Effect size for remission } & \mathbf{p} \\ \text { MA } & 50-400 \mathrm{mg} & 13 & \mathrm{RR}=1.24(1.16-1.32) & <0.001 & 12 & \mathrm{RR}=1.29(1.16-1.43) & <0.001 \\ \text { SA-1: WCS } & 50-400 \mathrm{mg} & 13 & \mathrm{RR}=1.24(1.16-1.32) & <0.001 & 12 & \mathrm{RR}=1.28(1.15-1.42) & <0.001 \\ \text { SA-1: BCS } & 50-400 \mathrm{mg} & 13 & \mathrm{RR}=1.24(1.16-1.32) & <0.001 & 12 & \mathrm{RR}=1.29(1.17-1.44) & <0.001 \\ \text { SA-2 } & 10-400 \mathrm{mg} & 13 & \mathrm{RR}=1.23(1.16-1.32) & <0.001 & 12 & \mathrm{RR}=1.27(1.15-1.40) & <0.001 \\ \text { SA-3 } & 50-400 \mathrm{mg} & 16 & \mathrm{RR}=1.26(1.19-1.34) & <0.001 & 15 & \mathrm{RR}=1.31(1.19-1.43) & <0.001 \\ \text { OR } & 50-400 \mathrm{mg} & 13 & \mathrm{OR}=1.48(1.32-1.66) & <0.001 & 12 & \mathrm{OR}=1.40(1.22-1.60) & <0.001 \\ \text { SA-4 } & 10-400 \mathrm{mg} & 13 & \mathrm{OR}=1.47(1.31-1.64) & <0.001 & 12 & \mathrm{OR}=1.37(1.20-1.57) & <0.001\end{array}$

BCS: best case scenario, MA: main analysis, N: number of trials included in the analysis, OR: odds ratio, RR: risk ratio, SA: sensitivity analysis, WCS: worst case scenario

Table 3 Risk ratio for responders for individual doses of desvenlafaxine.

\begin{tabular}{lccccr} 
& & \multicolumn{2}{c}{ Response } & \multicolumn{2}{c}{ Remission } \\
Dose & Trials & RR $(95 \%$ Cl) & P & RR (95\% Cl) & \multicolumn{1}{c}{ P } \\
\hline $50 \mathrm{mg}$ & 6 & $1.20(1.10-1.32)$ & $<0.001$ & $1.18(1.03-1.36)$ & 0.021 \\
$100 \mathrm{mg}$ & 5 & $1.27(1.15-1-41)$ & $<0.001$ & $1.40(1.20-1.63)$ & $<0.001$ \\
$200 \mathrm{mg}$ & 2 & $1.39(1.16-1.65)$ & $<0.001$ & $1.55(1.15-2.10)$ & 0.005 \\
\hline $400 \mathrm{mg}$ & 2 & $1.33(1.10-1.60)$ & 0.001 & $1.54(1.14-2.09)$ & 0.005 \\
Flexible & 4 & $1.20(1.04-1.38)$ & 0.012 & $1.23(0.97-1.55)$ & 0.083
\end{tabular}

RR: risk ratio, Cl: confidence intervals

\section{Risk of bias and publication bias}

The risk of bias for each study can be determined by assessing the following 6 domains: (1) sequence generation, (2) allocation concealment, (3) blinding, (4) missing data, (5) selective outcome reporting, and (6) other sources of bias. The overall risk of bias could be described as moderate ( $O$ Fig. 3 ). The results for the individual trials are presented in Appendix B. Finally, there is no indication of publication bias after visual inspection of the funnel plot; in particular, there is no gap on the bottom left side, which would be indicative of unpublished studies with small to moderate effects ( $\bullet$ Fig. 4). Egger's regression method also gave no indication of publication bias, since the intercept of the fitted line was near zero ( $\bullet$ Fig. 5 ).

\section{Discussion}

\section{Results}

The mean risk ratio for response was found to be 1.24 (95\% Cl: $1.16-1.32 ; p<0.001$ ), i.e., a therapeutic response is $25 \%$ more likely with the use of desvenlafaxine than in the placebo group, which can be regarded at best as a very moderate effect. Considering the fact that venlafaxine is currently one of the most effective antidepressants, this finding was quite unexpected. The head-to-head comparisons also provide some evidence that desvenlafaxine may be inferior when compared with other antidepressants. However, the robustness of these results is limited by the small number of included trials (only 4 ) and the heterogeneity of the population studied (one study included only peri- and postmenopausal women with depression). Further trials with direct comparisons are necessary in order to draw definite conclusions.

In our analysis there were no significant differences in the risk ratios for response and remission between the various doses (i.e., $50 \mathrm{mg}, 100 \mathrm{mg}, 200 \mathrm{mg}$ and $400 \mathrm{mg}$ ), although the 2 higher doses tended to have higher response rates. The lowest rates were found for the $50 \mathrm{mg}$ dose and the highest rates for the $200 \mathrm{mg}$ dose. 


$\begin{array}{lllllll}\mathbf{A} & \mathbf{B} & \text { Comparison } & \mathbf{R R d} & \mathbf{R R}_{\mathbf{A}} & \mathbf{R R}_{\mathbf{B}} & \mathbf{P} \\ 50 \mathrm{mg} & 100 \mathrm{mg} & \text { direct } & 0.97(0.87-1.09) & - & - & 0.622 \\ 50 \mathrm{mg} & 200 \mathrm{mg} & \text { indirect } & - & 1.20(1.10-1.32) & 1.37(1.15-1.64) & 0.202 \\ 50 \mathrm{mg} & 400 \mathrm{mg} & \text { indirect } & - & 1.20(1.10-1.32) & 1.34(1.12-1.61) & 0.292 \\ 100 \mathrm{mg} & 200 \mathrm{mg} & \text { indirect } & - & 1.24(1.12-1.38) & 1.37(1.15-1.64) & 0.354 \\ 100 \mathrm{mg} & 400 \mathrm{mg} & \text { indirect } & - & 1.24(1.09-1.41) & 1.34(1.12-1.61) & 0.466 \\ 200 \mathrm{mg} & 400 \mathrm{mg} & \text { direct } & 1.04(0.89-1.21) & - & - & 0.609\end{array}$

RRd: risk ratio for response when comparing direct dose $A$ against dose $B, R_{A}, R_{B}$ : risk ratio for response when comparing dose $A$ or dose $B$ with placebo

$\begin{array}{lllllll}\mathbf{A} & \mathbf{B} & \text { Comparison } & \mathbf{R R d} & \mathbf{R R}_{\mathbf{A}} & \mathbf{R R}_{\mathbf{B}} & \mathbf{P} \\ 50 \mathrm{mg} & 100 \mathrm{mg} & \text { direct } & 0.86(0.74-1.01) & - & - & 0.064 \\ 50 \mathrm{mg} & 200 \mathrm{mg} & \text { indirect } & - & 1.18(1.03-1.36) & 1.55(1.15-2.10) & 0.114 \\ 50 \mathrm{mg} & 400 \mathrm{mg} & \text { indirect } & - & 1.18(1.03-1.36) & 1.54(1.14-2.09) & 0.120 \\ 100 \mathrm{mg} & 200 \mathrm{mg} & \text { indirect } & - & 1.38(1.17-1.63) & 1.55(1.15-2.10) & 0.512 \\ 100 \mathrm{mg} & 400 \mathrm{mg} & \text { indirect } & - & 1.38(1.17-1.63) & 1.54(1.14-2.09) & 0.528 \\ 200 \mathrm{mg} & 400 \mathrm{mg} & \text { direct } & 1.01(0.78-1.30) & - & - & 0.962\end{array}$

RRd: risk ratio for remission when comparing direct dose $A$ against dose $B, R_{A}, R_{B}$ : risk ratio for remission when comparing dose $A$ or dose B with placebo

\begin{tabular}{|c|c|c|c|c|c|}
\hline \multirow[b]{2}{*}{ Comparison } & \multirow[b]{2}{*}{ Trials } & \multicolumn{2}{|c|}{ Response } & \multicolumn{2}{|c|}{ Remission } \\
\hline & & RR $(95 \%$ Cl) & $\mathbf{p}$ & $\operatorname{RR}(95 \% \mathrm{Cl})$ & $\mathbf{p}$ \\
\hline dVFX vs. VFX & 2 & $0.91(0.78-1.06)$ & 0.219 & $0.87(0.65-1.16)$ & 0.341 \\
\hline dVFX vs. SSNRIs & 3 & $0.92(0.81-1.04)$ & 0.168 & $0.85(0.70-1.05)$ & 0.126 \\
\hline dVFX vs. AD & 4 & $0.90(0.82-0.98)$ & 0.014 & $0.82(0.71-0.95)$ & 0.009 \\
\hline
\end{tabular}

\section{Table 6 Head-to-head} comparisons.

\section{Comparison with previous meta-analyses}

In a previous meta-analysis by Schueler et al. of 2 other selective serotonin and norepinephrine reuptake inhibitors, duloxetine and venlafaxine, the odds ratios for response compared with placebo were 1.99 (95\% CI: 1.65-2.39) and 2.04 (95\% CI: $1.74-$ 2.38 ), respectively, much higher than the OR for response of desvenlafaxine in our study ( $\mathrm{OR}=1.48,95 \% \mathrm{CI}: 1.32-1.66)$ [26]. Similarly, the odds ratio for remission was 1.40 (95\% CI: $1.22-$ $1.60)$ for desvenlafaxine, while the odds ratios for remission for duloxetine and venlafaxine were 1.91 (95\% CI: 1.56-2.34) and 1.97 (95\% CI: 1.64-2.35), respectively. As the confidence intervals of the odds ratio for response and remission do not overlap in the case of desvenlafaxine and venlafaxine, there appears to be a significant difference in their efficacy. Tolerability parameters were also provided in this meta-analysis; the odds ratios for discontinuation due to adverse events were 2.22 (95\% CI: $1.55-$ 3.19) for duloxetine and 2.47 (95\% CI: 1.81-3.37) for venlafaxine, while the odds ratio for desvenlafaxine in our meta-analysis was 2.07 (95\% CI: 1.48-2.89). In all, duloxetine and especially venlafaxine seem to have a better efficacy than desvenlafaxine, while tolerability of all 3 agents seems to be similar.

The above discrepancy in the odds ratios may reflect a true difference in the efficacies of desvenlafaxine and the other 2 SSNRIs, or alternatively can be attributed to factors related to the study design of the trials; for example multi-site and multi-arm trials can lead to an increased placebo effect; all desvenlafaxine trials were multi-site and 10 of the 12 studies in the main analysis were multi-arm $[27,28]$. It has also been mentioned that in more recent studies a higher placebo effect has been noticed in comparison to older ones [27]. Since desvenlafaxine is the newest drug of the 3 , this factor might also have played a role.
A recent meta-analysis performed an indirect comparison between desvenlafaxine and its parent substance and found no differences in their efficacy [29]. However, this study included only 7 trials with desvenlafaxine with a total of 2380 patients, about half the number included in our analysis. The authors did not report the risk ratios separately for each drug; when repeating our analysis using the population included in this indirect comparison, we found a risk ratio for response of 1.29 (95\% CI: $1.18-1.42, \mathrm{p}<0.001$ ) for desvenlafaxine, which is quite similar to our results. A non-significant difference between the 2 agents implies a similarly low efficacy for venlafaxine, which contradicts the results of the above meta-analysis by Schueler et al. In order to compare the results of all 3 studies, we estimated additionally the risk ratio for response to venlafaxine using the data provided in this latter meta-analysis; the results are presented in $\bullet$ Table 7. The confidence intervals of the risk ratios for venlafaxine overlap those for desvenlafaxine, as estimated both in the study by Coleman et al. and in our study. However, the odds ratios for response in our study in contrast to that by Coleman et al. appear to be significantly lower than the odds ratio for response for venlafaxine. Although this comparison is equivocal, it clearly demonstrates that it has not yet been established that the 2 agents are equally effective.

\section{Marketing active metabolites}

As mentioned above, the efficacy of active metabolites cannot be taken for granted. For example, norclozapine (desmethylclozapine or ACP-104) was ineffective in phase 2 trials in the treatment of schizophrenia, and further trials were not performed [30]. Similarly, the S-enantiomer of norfluoxetine (seproxetine), which is the main active metabolite of fluoxetine, did not qualify 


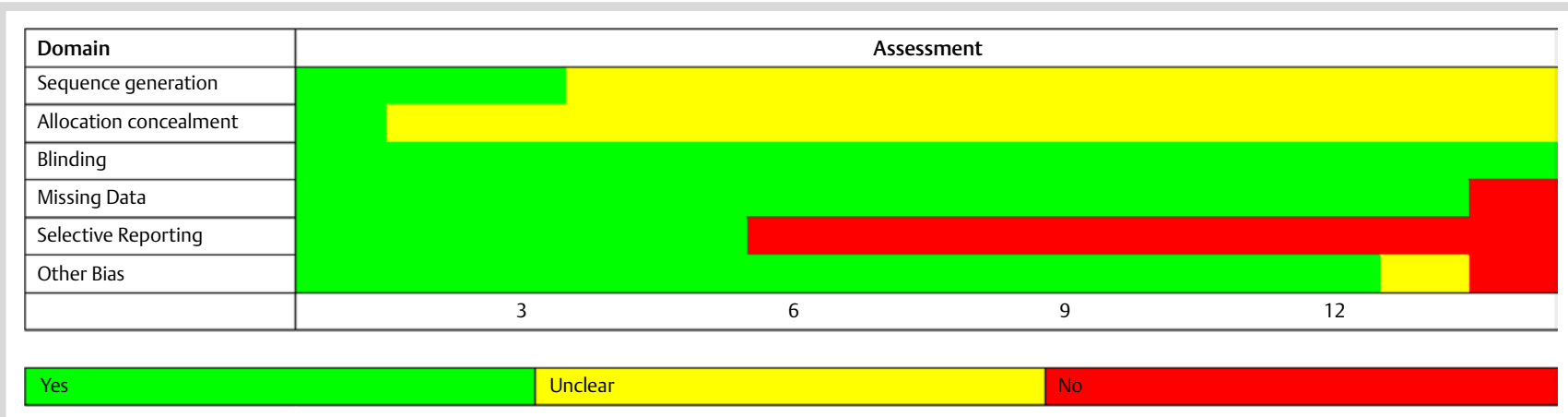

Fig. 3 Risk of bias graph. The semaphore colors provide a visual impression of the quality of the study reports for meta-analysis; green: condition is fulfilled; yellow: condition is questionable, and red: condition is not fulfilled and risk of bias is present. The overall risk for bias is moderate. (Color figure available online only).

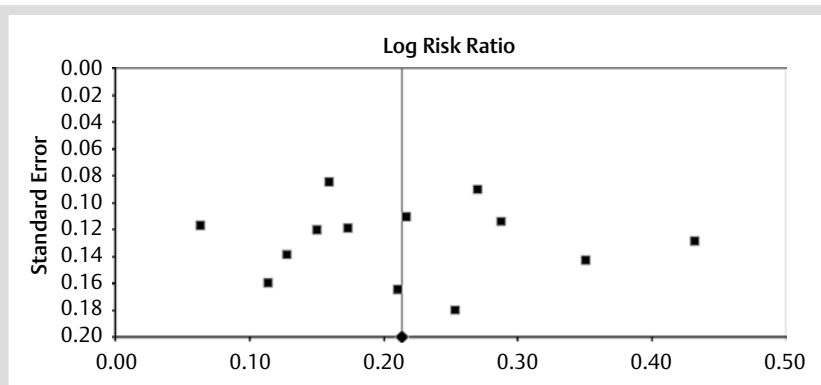

Fig. 4 Funnel plot. There is no gap on the bottom left that would be indicative of publication bias.

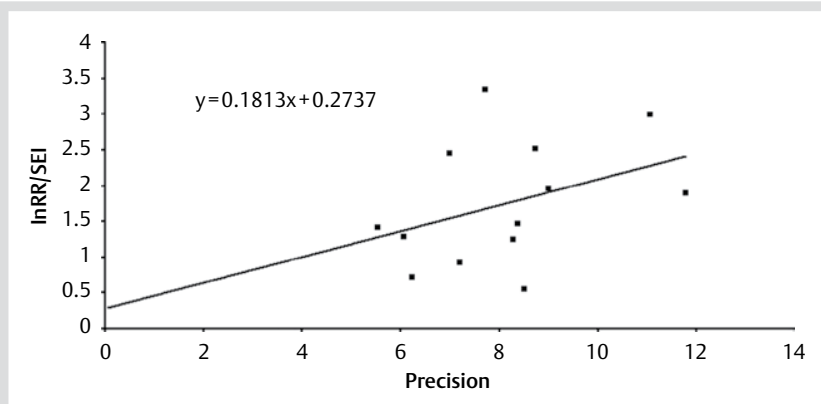

Fig. 5 Egger's regression method.

Table 7 Comparison of effect sizes for response for venlafaxine and desvenlafaxine.

\begin{tabular}{|c|c|c|c|}
\hline & $\begin{array}{l}\text { Current report } \\
\text { (dVFX) }\end{array}$ & $\begin{array}{l}\text { Coleman } \\
\text { et al. (dVFX) }\end{array}$ & $\begin{array}{l}\text { Schueler } \\
\text { et al. (VFX) }\end{array}$ \\
\hline $\begin{array}{l}\text { Risk ratio for } \\
\text { response }\end{array}$ & $1.24(1.16-1.32)$ & $1.29(1.18-1.42)$ & $1.41(1.30-1.52)$ \\
\hline $\begin{array}{l}\text { Odds ratio } \\
\text { for response }\end{array}$ & $1.48(1.32-1.66)$ & $1.62(1.36-1.92)$ & $2.04(1.74-2.38)$ \\
\hline
\end{tabular}

for phase 3 trials [31]. Leucht et al. showed in a recent metaanalysis that risperidone did not differ in either efficacy or safety parameters from its active metabolite paliperidone [3]. Consid- ering the fact that the active metabolites are much more expensive than the parent substances, whose patents have already expired, superiority or at least an equivalence of the former over the latter in terms of efficacy and tolerability should be demanded in order to justify their use.

\section{Limitations and strengths}

One limitation of this study is the inaccurate presentation of response and remission rates in the studies, requiring the estimation of approximate numbers of responders and remitters in the trials. However, sensitivity analysis showed that this approximation did not influence the results. Another limitation is that we extracted the number of patients with remission in one study by means of WebPlotDigitizer; although it has already been used in other medical studies, its accuracy has not yet been tested systematically. The strength of our report is the use of multiple sensitivity analyses, which allowed us to estimate the efficacy of desvenlafaxine in a relatively homogeneous population, while no information was lost since all trials were considered in at least one estimate of effect size.

\section{Conclusions}

In our meta-analysis the efficacy of desvenlafaxine was found to be moderate when compared to placebo. Direct comparisons to other antidepressants provide some evidence that desvenlafaxine might not be as efficient as other agents; however, these comparisons included only a small number of trials. Further head-to-head trials are necessary in order to draw definite conclusions. Based on the current literature we cannot support the view that desvenlafaxine should be used as a standard antidepressant agent; more evidence on its efficacy needs to be provided.

\section{Authors' Contributions \\ $\nabla$}

Zacharias G. Laoutidis conceived and designed the study, participated in data collection and evaluation, performed the statistical analysis and drafted the manuscript. Kanellos T. Kioulos participated in and supervised collection and analysis of data and helped to draft the manuscript. Both authors read and approved the manuscript. 


\section{Acknowledgements}

We would like to thank Prof. E. Angelopoulos for the useful discussions.

\section{Declaration of Interests}

$\nabla$

The authors declare no conflict of interest.

\section{References}

1 Cipriani A, Furukawa TA, Salanti G et al. Comparative efficacy and acceptability of 12 new-generation antidepressants: a multipletreatments meta-analysis. Lancet 2009; 373: 746-758 doi:10.1016/ S0140-6736(09)60046-5

2 Deecher DC, Beyer CE, Johnston $G$ et al. Desvenlafaxine succinate: A new serotonin and norepinephrine reuptake inhibitor. J Pharmacol Exp Ther 2006; 318: 657-665

3 Leucht S, Cipriani A, Spineli L et al. Comparative efficacy and tolerability of 15 antipsychotic drugs in schizophrenia: a multiple-treatments meta-analysis. Lancet 2013; 382: 951-962

4 Thase ME, Kornstein SG, Germain JM et al. An integrated analysis of the efficacy of desvenlafaxine compared with placebo in patients with major depressive disorder. CNS Spectr 2009; 14: 144-154

5 Higgins JPT, Green S. Cochrane Handbook for Systematic Reviews of Interventions. In The Cochrane Library, Volume Issue 4. Chichester, UK: John Wiley \& Sons, Ltd; 2006

6 Friedrich JO, Adhikari NKJ, Beyene J. Inclusion of zero total event trials in meta-analyses maintains analytic consistency and incorporates all available data. BMC Med Res Methodol 2007; 7: 5 doi:10.1186/14712288-7-5

7 Furukawa TA, Cipriani A, Barbui C et al. Imputing response rates from means and standard deviations in meta-analyses. Int Clin Psychopharmacol 2005; 20: 49-52

8 Borenstein M, Hedges RV, Higgins JPT et al. Introduction to MetaAnalysis.West Sussex: Wiley; Edition2009

9 Neyeloff JL, Fuchs SC, Moreira LB. Meta-analyses and forest plots using a Microsoft Excel spreadsheet: step-by-step guide focusing on descriptive data analysis. BMC Res Notes 2012; 5: 52 http://www.biomed central.com/1756-0500/5/52

10 Egger M, Davey Smith G, Schneider $M$ et al. Minder C. Bias in metaanalysis detected by a simple graphical test. BMJ 1997; 315: 629-634

11 DeMartinis NA, Yeung PP, Entsuah $R$ et al. A double-blind, placebocontrolled study of the efficacy and safety of desvenlafaxine succinate in the treatment of major depressive disorder. J Clin Psychiatry 2007; 68: 677-688

12 Liebowitz MR, Yeung PP, Entsuah R. A randomized, double-blind, placebo-controlled trial of desvenlafaxine succinate in adult outpatients with major depressive disorder.J Clin Psychiatry 2007; 68: 1663-1672

13 Septien-Velez L, Pitrosky B, Padmanabhan SK et al. A randomized, double-blind, placebo-controlled trial of desvenlafaxine succinate in the treatment of major depressive disorder. Int Clin Psychopharmacol 2007; 22: 338-347

14 Boyer $P$, Montgomery S, Lepola $U$ et al. Efficacy, safety, and tolerability of fixed-dose desvenlafaxine 50 and $100 \mathrm{mg} /$ day for major depressive disorder in a placebo-controlled trial. Int Clin Psychopharmacol 2008; 23: $243-253$

15 Lieberman DZ, Montgomery SA, Tourian KA et al. A pooled analysis of two placebo-controlled trials of desvenlafaxine in major depressive disorder. Int Clin Psychopharmacol 2008; 23: 188-197
16 Liebowitz MR, Manley AL, Padmanabhan SK et al. Efficacy, safety, and tolerability of desvenlafaxine $50 \mathrm{mg} /$ day and $100 \mathrm{mg} /$ day in outpatients with major depressive disorder. Curr Med Res Opin 2008; 24: 1877-1890

17 Feiger $A D$, Tourian KA, Rosas GR et al. A placebo-controlled study evaluating the efficacy and safety of flexible-dose desvenlafaxine treatment in outpatients with major depressive disorder. CNS Spectr 2009; 14: 41-50

18 Tourian KA, Padmanabhan SK, Groark J et al. Desvenlafaxine 50 and $100 \mathrm{mg} / \mathrm{d}$ in the treatment of major depressive disorder: an 8-week, phase III, multicenter, randomized, double-blind, placebo-controlled, parallel-group trial and a post hoc pooled analysis of three studies. Clin Ther 2009; 31: 1405-1423

19 Iwata $N$, Tourian KA, Hwang $E$ et al. Efficacy and safety of desvenlafaxine 25 and $50 \mathrm{mg} /$ day in a randomized, placebo-controlled study of depressed outpatients. J Psychiatr Pract 2013; 19: 5-14

20 Liebowitz MR, Tourian KA, Hwang $E$ et al. Study 3362 Investigators. A double-blind, randomized, placebo-controlled study assessing the efficacy and tolerability of desvenlafaxine 10 and $50 \mathrm{mg} /$ day in adult outpatients with major depressive disorder. BMC Psychiatry 2013; 13: 94

21 Clayton AH, Tourian KA, Focht $K$ et al. Desvenlafaxine 50 and $100 \mathrm{mg} / \mathrm{d}$ versus placebo for the treatment of major depressive disorder: a phase 4, randomized controlled trial. J Clin Psychiatry 2014 Oct 28. [Epub ahead of print]

22 Kornstein SG, Jiang $Q$ Reddy $S$ et al. Short-term efficacy and safety of desvenlafaxine in a randomized, placebo-controlled study of perimenopausal and postmenopausal women with major depressive disorder. J Clin Psychiatry 2010; 71: 1088-1096

23 Clayton AH, Kornstein SG, Dunlop BW et al. Efficacy and safety of desvenlafaxine $50 \mathrm{mg} / \mathrm{d}$ in a randomized, placebo-controlled study of perimenopausal and postmenopausal women with major depressive disorder. J Clin Psychiatry 2013; 74: 1010-1017

24 Dunlop BW, Reddy S, Yang $L$ et al. Symptomatic and functional improvement in employed depressed patients: a double-blind clinical trial of desvenlafaxine versus placebo. J Clin Psychopharmacol 2011; 31: $569-576$

25 Soares $C N$, Thase ME, Clayton A et al. Desvenlafaxine and escitalopram for the treatment of postmenopausal women with major depressive disorder. Menopause 2010; 17: 700-711

26 Schueler YB, Koesters $M$, Wieseler B et al. A systematic review of duloxetine and venlafaxine in major depression, including unpublished data. Acta Psychiatr Scand 2011; 123: 247-265

27 Papakostas GI, Fava M. Does the probability of receiving placebo influence clinical trial outcome? A meta-regression of double-blind, randomized clinical trials in MDD. Eur Neuropsychopharmacol 2009; 19: $34-40$

28 Bridge JA, Birmaher B, lyengar $S$ et al. Placebo response in randomized controlled trials of antidepressants for pediatric major depressive disorder. Am J Psychiatry 2009; 166: 42-49

29 Coleman KA, Xavier VY, Palmer TL et al. An indirect comparison of the efficacy and safety of desvenlafaxine and venlafaxine using placebo as the common comparator. CNS Spectr 2012; 17: 131-141

30 ACADIA Pharmaceuticals. ACADIA Pharmaceuticals Announces Results from ACP-104 Phase IIb Schizophrenia Trial. (Jun 16 2008); Internet http://news.acadia-pharm.com/phoenix.zhtml?c $=125180 \& \mathrm{p}=$ irolnewsArticle\&ID = 1166151

31 DrugBank 4.0: shedding new light on drug metabolism. Law V, Knox C, Djoumbou $Y$ et al. Nucleic Acids Res 2014; 42: D1091-D1097 PubMed ID: 24203711. Available from http://www.drugbank.ca/ drugs/DB06731\#references 


\section{Appendix}

\section{Appendix A Rejected studies.}

\section{Article}

Divyashree M, Jayanthi C, Chandrashekar $\mathrm{H}$. A comparative study of efficacy and safety of conventional vs. newer antidepressants in patients with depressive episode in a tertiary care hospital. J Chem Pharm Res. 2014;6:516-524

Soares CN, Fayyad RS, Guico-Pabia C]. Early improvement in depressive symptoms with desvenlafaxine $50 \mathrm{mg} / \mathrm{d}$ as a predictor of treatment success in patients with major depressive disorder. J Clin Psychopharmacol. 2014;34:57-65

Singh AP, Trivedi M, Singh Kushwah D. Comparative study of safety and efficacy of desvenlafaxine vs. sertraline: a randomized control trial. Int J Pharm Bio Sci 2014; 5:762-769

Cheng RJ1, Dupont C, Archer DF, Bao W, Racketa J, Constantine G, Pickar JH. Effect of desvenlafaxine on mood and climacteric symptoms in menopausal women with moderate to severe vasomotor symptoms. Climacteric. 2013;16:17-27 Ferguson JM1, Tourian KA, Rosas GR. High-dose desvenlafaxine in outpatients with major depressive disorder. CNS Spectr. 2012;17:121-30

\section{Reason for rejection}

Open label

Post hoc analysis

RCT. Included patients with mild to moderate depression. Depression did not belong to the eligible criteria.

Open label study.

Appendix B Assessment of bias. We used the Cochrane Collaboration's tool for assessing the risk of bias. These criteria may be considered sufficiently strict. Six domains were extracted and judged. The consensual authors' judgment was either "Yes," indicating low risk of bias, "No," indicating high risk of bias, or "Unclear," indicating unknown risk of bias. The criteria to assess the studies were:

\section{Domain}

Sequence generation

Allocation concealment

Blinding of participants, personnel, and outcome Incomplete outcome data

Selective outcome reporting

Other sources of bias

\section{DeMartinis, 2007}

Domain

Sequence generation

Allocation concealment

Blinding of participants,

personnel, and outcome

Incomplete outcome data

Selective outcome reporting

Other sources of bias

\section{Liebowitz, 2007}

Domain

Sequence generation

Allocation concealment

Blinding of participants,

personnel, and outcome

Incomplete outcome data

Selective outcome reporting

Other sources of bias

Septien-Velez, 2007

Domain

Sequence generation

Allocation concealment

Blinding of participants,

personnel, and outcome

Incomplete outcome data

Selective outcome reporting

Other sources of bias

\section{Description}

Describe the method used to generate the allocation sequence

Describe the method used to conceal the allocation sequence

Describe all measures used to blind participants and personnel

Describe the completeness of outcome data for each main outcome including attrition and exclusions from the analysis.

State how the possibility of selective outcome reporting was examined by the review authors and what was found.

State any important concerns about bias not addressed in the other domains.

\section{Description}

Randomized trial. Method is not described.

Assignment envelopes and drug containers are not described. Double blind trial.

The analysis is described as ITT, however it is actually an available case analysis.

For response and remission only the adjusted odds ratios were reported. Response and remission rates were not reported.

The study appears to be free of other sources of bias.

\section{Description}

Randomized trial. Method is not described.

Assignment envelopes and drug containers are not described. Double blind trial.

The analysis is described as ITT, however it is actually an available case analysis.

P-values are not reported for all the results, especially when insignificant.

The study appears to be free of other sources of bias.

\section{Description}

Randomized trial. Method is not described.

Assignment envelopes and drug containers are not described. Double blind trial.

The analysis is described as ITT, however it is actually an available case analysis.

All prespecified outcomes of interest are reported in the prespecified way.

The study appears to be free of other sources of bias.

\section{Review Author's Judgement}

Was the allocation sequence adequately generated? (Yes, No, Unclear)

Was allocation adequately concealed? (Yes, No, Unclear) Was knowledge of the allocated intervention adequately prevented during the study? (Yes, No, Unclear)

Were incomplete outcome data adequately addressed? (Yes, No, Unclear)

Are reports of the study free of suggestion of selective outcome reporting? (Yes, No, Unclear)

Was the study apparently free of other problems that could put it at high risk of bias?

Review Author's Judgement

Unclear.

Unclear.

Yes.

Yes.

No.

Yes.

Review Author's Judgement

Unclear.

Unclear.

Yes.

Yes.

No.

Yes.

Review Author's Judgement

Unclear.

Unclear.

Yes.

Yes.

Yes.

Yes. 
Appendix B Continued.

Domain

Boyer, 2008

Domain

Sequence generation

Allocation concealment

Blinding of participants,

personnel, and outcome

Incomplete outcome data

Selective outcome reporting

Other sources of bias

Lieberman, 2008

Domain

Sequence generation

Allocation concealment

Blinding of participants,

personnel, and outcome

Incomplete outcome data

Selective outcome reporting

Other sources of bias

\section{Liebowitz, 2008}

Domain

Sequence generation

Allocation concealment

Blinding of participants, personnel, and outcome Incomplete outcome data

Selective outcome reporting

Other sources of bias

\section{Feiger, 2009}

Domain

Sequence generation

Allocation concealment

Blinding of participants,

personnel, and outcome

Incomplete outcome data

Selective outcome reporting

Other sources of bias

Tourian, 2009

Domain

Sequence generation

Allocation concealment

Blinding of participants,

personnel, and outcome

Incomplete outcome data

Selective outcome reporting

Other sources of bias

\section{Kornstein, 2010}

Domain

Sequence generation

Allocation concealment

Blinding of participants,

personnel, and outcome

Incomplete outcome data

Selective outcome reporting

\section{Description}

Description

Randomized trial. Method is not described.

Assignment envelopes and drug containers are not described. Double blind trial.

The analysis is described as ITT, however it is actually an available case analysis.

All prespecified outcomes of interest are reported in the prespecified way.

The study appears to be free of other sources of bias.

Description

Randomized trial. Method is not described.

Assignment envelopes and drug containers are not described.

Double blind trial.

The analysis is described as ITT, however it is actually an available case analysis.

Discontinuation rates and reasons for discontinuation are not reported.

The study appears to be free of other sources of bias.

Description

Randomized trial. Block randomization schedule. Block size was $6(2: 2: 2)$

Assignment envelopes and drug containers are not described. Double blind trial.

The analysis is described as ITT, however it is actually an available case analysis.

Response and remission rates were not reported.

The study appears to be free of other sources of bias.

Description

Randomized trial. Method is not described.

Assignment envelopes and drug containers are not described.

Double blind trial.

The analysis is described as ITT, however it is actually an available case analysis.

All prespecified outcomes of interest are reported in the prespecified way.

The study appears to be free of other sources of bias.

Description

Randomized trial. Block randomization schedule. Block size was $8(2: 2: 2: 2)$.

Central allocation.

Double blind study. No indications that blinding could have been broken.

The analysis is described as modified ITT, which is actually an available case analysis. LOCF

No $p$-values are provided for the response and remission rates.

The study appears to be free of other sources of bias.

Description

Central computerized randomization system.

Not described.

Double blind trial.

Modified ITT: results only from a subgroup of the sample. They report only a subgroup of the sample.
Review Author's Judgement

Review Author's Judgement

Unclear.

Unclear.

Yes.

Yes.

Yes.

Yes.

Review Author's Judgement

Unclear.

Unclear.

Yes.

Yes.

No.

Yes.

Review Author's Judgement

Yes.

Unclear.

Yes.

Yes.

No.

Yes.

Review Author's Judgement

Unclear.

Unclear.

Yes.

Yes.

Yes.

Yes.

Review Author's Judgement

Yes.

Yes.

Yes.

Yes.

No.

Yes.

Review Author's Judgement

Yes.

Unclear.

Yes.

No.

No. 
Appendix B Continued.

\section{Domain}

Other sources of bias

Dunlop, 2011

Domain

Sequence generation Allocation concealment Blinding of participants, personnel, and outcome Incomplete outcome data

Selective outcome reporting

Other sources of bias

Clayton, 2013

Domain

Sequence generation

Allocation concealment

Blinding of participants, personnel, and outcome

Incomplete outcome data

Selective outcome reporting

Other sources of bias

Iwata, 2013

Domain

Sequence generation

Allocation concealment

Blinding of participants, personnel, and outcome Incomplete outcome data

Selective outcome reporting

Other sources of bias

Liebowitz, 2013

Domain

Sequence generation

Allocation concealment

Blinding of participants, personnel, and outcome Incomplete outcome data

Selective outcome reporting

Other sources of bias

Soares, 2010

Domain

Sequence generation

Allocation concealment

Blinding of participants,

personnel, and outcome

Incomplete outcome data

\section{Description}

The participants were enrolled based on their MADRS score, but the efficacy is estimated based on the HAMD score. The authors then use a subgroup of the sample with HAMD $>18$ for the estimation of efficacy and ignore the rest of the sample.

\section{Description}

Randomized trial. Method is not described.

Not described.

Double blind trial.

The analysis is described as ITT, however it is actually an available case analysis. LOCF.

All prespecified outcomes of interest are reported in the prespecified way.

The study appears to be free of other sources of bias.

Description

Randomized trial. Method is not described.

Assignment envelopes and drug containers are not described. Double blind trial.

The analysis is described as ITT, however it is actually an available case analysis. LOCF.

All prespecified outcomes of interest are reported in the prespecified way.

The participants were enrolled based on their MADRS score, but the efficacy is estimated based on the HAMD score. The authors then use a subgroup of the sample with HAMD $>18$ for the estimation of efficacy. It is unclear, if this method biases the results.

\section{Description}

"Study site personnel called an automated system to receive a subject randomization number and a package number."

"Study site personnel called an automated system to receive a subject randomization number and a package number." Double blind trial.

The analysis is described as ITT, however it is actually an available case analysis. LOCF.

All prespecified outcomes of interest are reported in the pre-specified way. P-values from non-significant results are missing.

The study appears to be free of other sources of bias.

Description

"Study site personnel called an automated system to receive a subject randomization number and a package number."

"Study site personnel called an automated system to receive a subject randomization number and a package number." Double blind trial.

The analysis is described as ITT, however it is actually an available case analysis. LOCF.

All prespecified outcomes of interest are reported in the pre-specified way. P-values from non-significant results are missing.

The study appears to be free of other sources of bias.

\section{Description}

Computerized and randomization system.

Details are not provided.

Double blind trial.

Modified ITT: results only from a subgroup of the sample.
Review Author's Judgement

No.

Review Author's Judgement

Unclear.

Unclear.

Yes.

Yes.

Yes.

Yes.

Review Author's Judgement

Unclear.

Unclear.

Yes.

Yes.

Yes.

Unclear.

Review Author's Judgement

Unclear.

Unclear.

Yes.

Yes.

No.

Yes.

Review Author's Judgement Unclear.

Unclear.

Yes.

Yes.

No.

Yes.

Review Author's Judgement

Yes.

Unclear.

Yes.

No. 
Appendix B Continued.

\begin{tabular}{|c|c|c|}
\hline Domain & Description & Review Author's Judgement \\
\hline Selective outcome reporting & They report only a subgroup of the sample. & No. \\
\hline Other sources of bias & $\begin{array}{l}\text { The participants were enrolled based on their MADRS score, } \\
\text { but the efficacy is estimated based on the HAMD score. The } \\
\text { authors then use a subgroup of the sample with HAMD }>18 \text { for } \\
\text { the estimation of efficacy and ignore the rest of the sample. }\end{array}$ & No. \\
\hline \multicolumn{3}{|l|}{ Clayton, 2014} \\
\hline Domain & Description & Review Author's Judgement \\
\hline Sequence generation & Randomized trial. Randomization procedure is not described. & Unclear. \\
\hline Allocation concealment & Assignment envelopes and drug containers are not described. & Unclear. \\
\hline $\begin{array}{l}\text { Blinding of participants, } \\
\text { personnel, and outcome }\end{array}$ & Double blind trial. & Yes. \\
\hline Incomplete outcome data & $\begin{array}{l}\text { The analysis is described as ITT, however it is actually an avail- } \\
\text { able case analysis. }\end{array}$ & Yes. \\
\hline Selective outcome reporting & $\begin{array}{l}\text { All prespecified outcomes of interest are reported in the } \\
\text { pre-specified way. P-values from non-significant results are } \\
\text { missing. }\end{array}$ & No. \\
\hline Other sources of bias & The study appears to be free of other sources of bias. & Yes. \\
\hline
\end{tabular}

